International Journal of Modern Physics: Conference Series

(C) World Scientific Publishing Company

\title{
NONCOMMUTATIVITY AND HUMANITY - JULIUS WESS AND HIS LEGACY
}

\author{
GORAN S. DJORDJEVIC \\ Department of Physics, Faculty of Science and Mathematics, University of Niš \\ P.O. Box 224, 18000 Niš, Serbia \\ gorandj@junis.ni.ac.rs
}

Received 10 March 2012

\begin{abstract}
A personal view on Julius Wess's human and scientific legacy in Serbia and the Balkan region is given. Motivation for using noncommutative and nonarchimedean geometry on very short distances is presented. In addition to some mathematical preliminaries, we present a short introduction in adelic quantum mechanics in a way suitable for its noncommutative generalization. We also review the basic ideas and tools embedded in $q$-deformed and noncommutative quantum mechanics. A rather fundamental approach, called deformation quantization, is noted. A few relations between noncommutativity and nonarchimedean spaces, as well as similarities between corresponding quantum theories, in particular, quantum cosmology are pointed out. An extended Moyal product in a frame of an adelic noncommutative quantum mechanics is also considered.
\end{abstract}

Keywords: Noncommutativity; nonarchimedean spaces; Moyal product; $p$-adic analysis; quantum cosmology.

PACS numbers: 01.60.+q, 01.78.+p, 02.40.Gh, 98.80.-k, 98.80.Qc

\section{Julius Wess - Scientist and Humanist}

The role of Julius Wess in the renewal and development of research in the field of high energy physics, especially in Serbia and Croatia, but also in other parts of former Yugoslavia and beyond, and in particular in what we shall refer to as international cooperation, is significant and large. This positive influence can be felt even now and will continue although he has passed away. The significance of his human and scientific mission in this part of the world is not well enough known in many respects. That is even in Germany and in the institutions in which he worked and which he managed in those years, and from which he drew the logistic, financial and every other form of support for this mission, above all the Max Planck Institute (MPI) for Physics and Ludwig Maximilian University (LMU) Munich. In one segment of this paper I shall attempt, from my own point of view and on the basis of my knowledge and memories, to shed light on the period of our friendship and cooperation in the period from 2000 to 2007. It is my desire that this aspect of his noble personality and immaterial legacy be preserved for remembrance as a 
valuable example for the future, as well as to provide an overview of the processes which were initiated thanks to Julius Wess and which continue to this day.

\subsection{Initial information and contacts}

Much can be learned about the process of the forming of the idea and beginnings of Julius' initiative Scientists in Global Responsibility (Wissenschaftler in Global Verantwortung - WIGV in German), from a valuable text in this volume by Lutz Möller, 1 who was a student of Julius', and also a very important associate in the period from 1999 to 2003.

The first information about the WIGV initiative and two postdoctoral scholarships in Munich in the field of theoretical physics and mathematics for candidates from former Yugoslavia reached me early in March of 2000 through Branko Dragović (my supervisor in the period from 1989 to 1999), thanks to his teaching engagement at the University of Banja Luka and the information he received there. By the time I had made first contact and applied (with the recommendation of B. Dragović), which went via Martin Schottenloher, Julius' colleague from the Department of Mathematics at LMU, both scholarships had been awarded to two mathematicians. Julius' desire to support as many young people as possible, his influence and ability to quickly find the most favorable solution, led to a sequence of events which, following my first lecture given early in July in Munich, resulted in my receiving the position of guest researcher, which lasted, with short breaks, almost two years, until the end of November 2002.

\subsection{WIGV and scholarships}

Scholarships for students and researchers from former Yugoslavia were probably one of the most significant segments of Julius' contribution to the improvement of research in the region, above all in the field of theoretical physics, as well as mathematics.

As early as 1999, Igor Baković from the Faculty of Mathematics and Institute Rudjer Bošković in Zagreb received a full scholarship for a doctorate in the field of applications of noncommutative geometry on short distance physics. As has already been mentioned, in the course of 2000, as part of a special DFG program (Noncommutative fields in physics) two postdoctoral scholarships were given to Svjetlana Terzić from Podgorica and Božidar Jovanović from Belgrade. In addition to myself, somewhat later, and funded from other sources, mobility grants to Munich were awarded to Goran Duplančić, Josip Trampetić, Dragutin Svrtan and Larisa Jonke from Zagreb, Marija Dimitrijević, Maja Burić and Voja Radovanović from Belgrade. In those years, numerous researchers stayed in Munich from Montenegro, Bulgaria, Ukraine, Russia, Morocco etc.

Almost all scholarships were realized via the LMU and/or MPI for Physics in Munich. Julius thus made it possible, especially but not exclusively, for younger 
researchers to spend significant time working and improving their research capacities under very favorable circumstances in his group.

A range of other programs and conferences which he led or organized (especially workshops in Bayrischzell) which were continued even after his death, made possible meetings and cooperation between numerous researchers from the region. Maybe most significantly, he made possible to us to establish contacts and cooperation with many excellent researchers from all around the world, who were in various ways linked to Julius and who visited Munich.

In addition to the positive effect on the scientific orientation and therefore the careers of the researchers named and those unnamed, as complete and precise documentation is not available, these programs had a range of indirect, sometimes no less significant effects. Knowledge was transferred not only to individuals but to the institutions where researchers were employed. it made it possible for their students and colleagues to be a part research international programs and to cooperate with many other institutions in Germany, Europe and around the world.

Likewise, new contacts were established and some others reinvigorated between individuals and scientific centers in the Balkans region. We emphasize this region as the text deals mainly with the influence which Julius in the last count achieved in the Balkans, that is, in Southeast Europe (SEE), although the term SEE is geographically not entirely correct.

\subsection{The organization of conferences in the region}

It must be emphasized that in Serbia, focussing for a moment only on this republic of the former Yugoslavia, for at least one whole decade, from 1991 to 2001, not a single international conference had been held, no school, workshop or any other form of scientific meeting, in the field of theoretical physics and mathematical physics on high energies. Researchers and students in Serbia were practically without the possibility of participating in similar meetings abroad, not to mention in the West. Therefore, Julius' idea, launched in October 2000 for organizing the first German-Serbian school of modern mathematical physics was of great importance. The summer school was held in Sokobanja, Serbia (in August of 2001), and it was much more than yet another Summer School somewhere in Europe.

The School was organized and took place an effort that was truly pioneering and with a great desire that after a horrible decade in former Yugoslavia, in all areas of life as well as in physics. The aim was to make it possible for students, above all, to attend an international school of mathematical physics of the highest level, with the participation of a larger number of lecturers and students from different countries. Main financial support came only two days before the beginning of the School, thanks to Julius' efforts and standing which he had with the Bundesministerium für Bildung and Forschung (Federal Ministry of Education and Research - Germany). The school had 65 participants, 13 lecturers had cycles of three to four lectures, while another 13 had lectures which were closer in form to workshops. 
Although Julius was unable to attend the School, seven students from Germany participated in it, above all his $\mathrm{PhD}$ students from Munich. For me, as someone who had grown up in a different culture, it took time and trust to believe they had come to Serbia so soon after all the wars, led only by own free will, attracted either by the program offered and recommended by their supervisor. This was a pleasant and valuable discovery. In addition to a varied program of science and teaching, there was a social program, and the socializing had an irreplaceable positive effect on later closeness, trust and cooperation between colleagues from different countries.

The organization and success of the School, in addition to the great roles of Julius Wess and Lutz Möller were in large part also due to the efforts of the Institute for Physics in Belgrade, above all Branko Dragović and Dragan Popović, as well as my colleagues and associates from the Department of Physics of the University of Niš, Ljubiša Nešić, Jelena Stanković and Dragoljub Dimitrijević.

Although at some moments in the preparation it seemed as if the organization of the entire 12-day School hung by a thread, and communication among organizers from different countries, cultures and mentalities, who were practically cooperating for the first time in this way, was put to the test, the impression that the School was very successful was confirmed only a few days after its completion at the very next 8th Adriatic meeting held in Dubrovnik, Croatia.

The story of the Adriatic meeting is beyond the immediate framework of the subject, however, it should be mentioned that the Adriatic meeting in the 1970s and 1980s was an international scientific conference of the highest level which was so significant that it was clearly noted beyond the boundaries of former Yugoslavia. I believe that nostalgia for the good old times and conferences which Julius himself had attended on several occasions at a time when tensions between the different peoples of former Yugoslavia were not felt and noticed as much, was a motive for Julius to place the revival of the Adriatic meeting, in addition to other programs, high on the list of priorities of the WIGV initiative. In brief, Adriatic meeting 2001 was an excellent, valuable conference, excellently organized by colleagues from Croatia led by Josip Trampetić.

It is significant for our topic that in conversations with Julius and colleagues from Croatia (J. Trampetić, N. Bilić, B. Guberina and others) it was agreed that the second German-Serbian school of modern mathematical physics should be held again in 2002 in Serbia (not in 2003 as previously agreed), so that the School (focussed on mathematical physics) and Adriatic meeting (with an accent on phenomenology in high energy physics) could be organized in even or uneven years respectively, and in this way could address the need of both the training of young researchers and exchange of knowledge and achievements among leading researchers.

A situation in which Serbian and Croatian physicists talk with an AustrianGerman physicist, support one another, find individual and shared interests which are almost identical, was for me, as someone new to these types of activities, something of a novelty and very encouraging. This was probably the decisive influence for the idea of forming some kind of network (which would later receive its acronym 
SEENET-MTP). It should also be mentioned that the later development of events would show that we were all a bit too optimistic about the range and speed of revival of scientific life in our region.

Early in 2002, within the inner circle of physicists in Serbia there came about a disagreement about the organization of the Second School and the need for and ways of forming a regional network in Mathematical and Theoretical Physics. In the next four to five years this would lead to a separation in the organization of the 2nd and 3rd and partially the 4th School of Mathematical Physics on the one hand, and the founding of the SEENET-MTP network and its meetings, above all BW2003, BW2005, BW2007 etc. Julius, in accordance with his health, which took a dramatic turn for the worse in 2003, and opportunities for influence after retiring, assisted all the above meetings and activities greatly. He also supported a few meetings in pure Mathematics in the region.

\subsection{Forming of the SEENET-MTP Network}

This text is not a text on the SEENET-MTP network, but on Julius' contribution to the revival of scientific life and cooperation in some fields of science in the Balkans, hence only some basic data will suffice.

Staying and working in Munich, in Julius' group, at the LMU and MPI was a fantastic experience. Although I already had some experience in a rich program of seminars and lectures given by great scientists at the Mathematics Institute Steklov in Moscow, a new quality appeared here. Guest lecturers were most often connected to corresponding groups in Munich through international projects and networks of institutions and research groups. Julius' wish was to help, as much as possible, in connecting scientists from former Yugoslavia. My impression was that earlier, and in particular in 2002, a "critical mass" of students and researchers in this region could not have been achieved and that a larger context should be attempted - the Balkans, which would include Turkey, Greece, Bulgaria, Romania etc. In my belief this kind of approach would in addition to a scientific also have a political dimension and other numerous benefits. Agreement was quickly reached and the name Southeastern European Network in Mathematical and Theoretical Physics (SEENET-MTP, www.seenet-mtp.info) was created in an informal atmosphere ... in the triangle Wess-Trampetić-Djordjević, in the vicinity of Alta and Neue Pinakotek, in Munich, sometime early in July of 2002. With Julius' personal recommendations in this period I visited CERN, ICTP, UNESCO headquarters in Paris and the UNESCO Office for Europe in Venice. In the course of these visits and talks the foundations were laid for support for the future SEENET-MTP network. Let mention just a few the most supportive persons from UNESCO, Dr. Vladimir Kouzminov, Dr. Maciej Nalecz and Dr. Vladimir Zharov.

The founding meeting of the network was envisaged as a workshop Balkan Workshop - BW2003, devoted to Mathematical, Theoretical and Phenomenological Challenges Beyond the Standard Model, with the addition of Perspectives of Balkans 
Collaboration and as a satellite meeting the Fifth General Conference of the Balkan Physical Union (Vrnjačka Banja, Serbia, August 2003). This made it possible for the meeting to have a regional dimension in many respects, to have present representatives of practically all countries from the region and to secure savings, in particular in travel expenses. Unlike the First School and some other actions the contribution to the budget of BW2003 from Germany was not more than one third. Significant contributions came from DFG (with Julius' support) and DAAD (with the support of Dieter Lüst). It was for us a very difficult but important schooling in the writing of several applications, projects and their implementation. This scientific meeting with excellent lecturers was completed with the ratification of a letter of intent, 2 my election as Coordinator of the Network and Julius' election as Coordinator of the Network's Scientific Advisory Committee (SAC).

While singling out the role of an individual might seem disproportionate, the role of Boyka Aneva in motivating colleagues and institutions from Sofia and Mihai Visinescu for those from Romania was crucially important.

The publishing of the Proceedings of BW2003, which consisted of almost all invited lectures, was a formidable task for us. Without Julius' authority this endeavor would not have been successful. With the joint efforts of the editors, authors and volunteers from Niš, the Proceedings 3 was published in cooperation with World Scientific and found its place in libraries around the world and was the scientific ticket and identity card of the SEENET-MTP network and its scientific potential in the next few years.

Less than two months after BW2003 Julius had a serous cardiovascular accident in Vienna and our first meeting after that was in Munich in January 2004. We spent more time discussing my fight with stress than his condition, operation and almost miraculous recovery in a relatively short period of time! It has been a great pleasure to mention that early in November of 2003, by the proposal of several physicists from Serbia, Julius was elected a member of the Serbian Academy of Science and Arts.

From a range of joint activities over the next years, I would like to single out his participation at the, small in terms of number of participants but significant, workshop Quantum Models - QM2005, which was held in Niš in November 2005. At this meeting the structure of the SEENET-MTP network was laid out and its Terms of References were ratified as the main regulatory act for the functioning of the network until the present day.

All scientific meetings in the period from 2003 to 2007 were organized with Julius' help, despite his health problems, about which he almost never complained. All scientific publications, above all proceedings, were prepared and published under his expert guidance. Several projects which we realized with UNESCO were also joint efforts with Julius. Adding all exchange programs in this period, of course without counting conferences, around 50 stays of scientists at foreign institutions in South East Europe could be realized in the framework of SEENET-MTP, as well as a number of outstanding guest lecturers from Western Europe and the USA 
With the ending of the Balkan Summer Institute BSI2011, one of the four segments of which was a Workshop devoted to the Scientific and Human Legacy of Julius Wess, SEENET-MTP has from the outset 50 individual founders and three institutions (Bucharest, Sofia, Niš) grown to a network of 19 institutions in ten countries of the Balkans (only Montenegro is missing), with around 300 individual members and 12 partner institutions from around the world. With some ten projects realized with support from UNESCO, ICTP, Bavarian Ministry for Science and other institutions, and a similar number of scientific meetings, now not only in Serbia and Croatia, but also in Bulgaria, Romania, Turkey, and several joint monographs and proceedings, as well as many individual and joint papers referring to the SEENET-MTP (and related projects), despite all the weakness and problems, we can say that we have much more than we had in 2000. A large gap has been left with Julius' passing but has been somewhat made up by the activities of the new coordinator of SAC Goran Senjanović, the president of the Network and its Representative Committee as of 2009, Radu Constantinescu and Michael Haack from LMU Munich in many ways. Some one hundred students who have passed through these programs and activities, we hope, will go on to raise the level of scientific research in the region to a higher one than our generation has been able to achieve. In every one of these successes will be a part of Julius' idea from 1999 and a part of his personal benevolence.

\subsection{In place of a conclusion}

Julius Wess has in many respects influenced scientific life and the work of a significant number of physicists and mathematicians from former Yugoslavia in changing and improving it at a moment when it seemed that there was no help coming and when our societies had fallen into some forms of poverty worse than just material. In addition to direct financial support (SINYu/SINSEE - modern intranet for the Faculties of Science in Belgrade, Niš and Novi Sad, and access to high speed internet as an investment of around one million Euros, the greatest donation in books for physics in Niš ever, in addition to similar donations in Tuzla and Banja Luka 1 , great moral motivation to begin and persevere with a unique project of the High school class for students with special abilities in physics in Niš, $\frac{4}{4}$ his suggestions and proposals have opened the doors of many institutions and foundations for individual as well as strategic programs.

Perhaps most importantly, he transferred to us his view of physics, his way of thinking and doing research, encouraged us to address serious problems, to approach great scientists and with his personal example, taught us to be humble with and approachable for those younger and not as well known.

In the end, a special personal note, his warm attitude, and the attitude of his wife Traudi, towards my family and in particular the children is something I will always remember. 


\section{Noncommutative vs Nonarchimedean Geometry at Short Distances - Motivation}

It is widely accepted that the standard picture of space-time should be changed around and beyond Planck scale. "Measuring" of spacetime geometry under distances smaller than Planck length $l_{p}$ is not accessible even to Gedanken experiments. It serves the idea of "quantization" and "discretization" of spacetime and a natural cutoff when using a quantum field theory to describe related phenomena. We are pointing out two possibilities for a reasonable mathematical background of a quantum theory on very small distances. The first one comes from the idea of spacetime coordinates as noncommuting operators 5

$$
\left[\hat{x}^{i}, \hat{x}^{j}\right]=i \theta^{i j} .
$$

Some noncommutativity of configuration space should not be a surprise in physics since quantum phase space with the canonical commutation relation

$$
\left[\hat{x}^{i}, \hat{k}^{j}\right]=i \hbar \delta^{i j},
$$

where $x^{i}$ are coordinates and $k^{j}$ are the corresponding momenta is the well-known example of noncommutative (pointless) geometry. This relation is connected in a natural way with the uncertainty principle and a "fuzzy" spacetime picture at distances $\theta^{1 / 2}$. Although it seems to make good physical sense for $\theta^{1 / 2} \sim l_{n}$, characteristic noncommutative distances could be related to gauge couplings $\frac{[5]}{,}$ closer to observable distances. It should be noted that the deriving of uncertainty relation $\left(\delta x>l_{p}\right)$ leads to a "strange" notion of quantum line and probably beyond archimedean geometry, because a coordinate always commutes with itself 6

The second promising approach to physics at the Planck scale, based on nonarchimedean geometry, was suggested 7 The simplest way to describe such a geometry (often referred to as ultrametric) is by using $p$-adic number fields $\mathbb{Q}_{p}$ ( $p$ is a prime). On the basis of the Ostrowski theorem 8 there are no other nontrivial possibilities (besides the field of real numbers $\mathbb{R}$ ) to complete the field of rational numbers $\mathbb{Q}$ with respect to a (nontrivial) norm.

There have been many interesting applications of $p$-adic numbers and nonArchimedean geometry in various parts of modern theoretical and mathematical physics (for a review, see Refs. 8, 9, 10). However, we restrict ourselves here mainly to $p$-adic 11 and adelic 12 quantum mechanics (QM). It should be noted that adelic QM have appeared quite useful in quantum cosmology $13[14$ The appearance of space-time discreteness in adelic formalism (see, e.g. Ref. 13), as well as in noncommutative QM, is an encouragement for further investigations. The $p$-adic analysis and noncommutativity also play a role in some areas of "macroscopic" physics 15

The interplay between noncommutative and nonarchimedean approach to quantum theory on very short distances was the main topic of my research during "Munich period" and subject of numerous discussions with Julius and his collaborators. This investigation continues to be among my main research topics till now. 
This paper is organized as follows: a personal view on Julius Wess human and scientific legacy in Serbia and Balkan region is followed by this note about motivation for investigation on a possible role of noncummatative and nonarchimedean geometry in physics. A short introduction to $p$-adic numbers, adeles and their functions is given in section 3 . This section is completed by a review of $p$-adic and adelic QM based on the Weyl quantization and Feynman's path integral. Previously observed and originally discovered relations between noncommutative and $p$-adic QM are discussed in section 4. A particular result on a new observed relation between an ordering on commutative ring in frame of deformation quantization 1617 and an ordering on $p$-adic spaces with the intention to develop path integration on $p$-adics by "slicening" of trajectories 18 is emphasized. Section 5 is devoted to a proposal for formulation of noncommutative adelic QM and some aspects of the extended Moyal product! 19 This paper closes with a presentation of an application of commutative and noncommutative mechanics in cosmology on real and $p$-adic spaces with notes on some ideas for further investigation, in particular, towards tachyonic cosmological (inflatory) models. 20

\section{3. -Adic Numbers, Adeles and Quantum Mechanics}

Any $p$-adic number $x \in \mathbb{Q}_{p}$ can be presented in the form 8

$$
x=p^{\nu}\left(x_{0}+x_{1} p+x_{2} p^{2}+\cdots\right), \quad \nu \in \mathbb{Z},
$$

where $x_{i}=0,1, \cdots, p-1$ are digits and $p$ is a prime number. $p$-Adic norm of any term $x_{i} p^{\nu+i}$ in the canonical expansion (3) is $\left|x_{i} p^{\nu+i}\right|_{p}=p^{-(\nu+i)}$ and the strong triangle inequality holds, i.e. $|a+b|_{p} \leq \max \left\{|a|_{p},|b|_{p}\right\}$. It follows that $|x|_{p}=p^{-\nu}$ if $x_{0} \neq 0$. Derivatives of $p$-adic valued functions $\varphi: \mathbb{Q}_{p} \rightarrow \mathbb{Q}_{p}$ are defined as in the real case, but with respect to the $p$-adic norm. There is no integral $\int \varphi(x) d x$ in a sense of the Lebesgue measure, $\frac{8}{6}$ but one can introduce $\int_{a}^{b} \varphi(x) d x=\Phi(b)-\Phi(a)$ as a functional of analytic functions $\varphi(x)$, where $\Phi(x)$ is an antiderivative of $\varphi(x)$. In the case of map $f: \mathbb{Q}_{p} \rightarrow \mathbb{C}$ there is well-defined Haar measure. One can use the Gauss integral

$$
\int_{\mathbb{Q}_{v}} \chi_{v}\left(a x^{2}+b x\right) d x=\lambda_{v}(a)|2 a|_{v}^{-\frac{1}{2}} \chi_{v}\left(-\frac{b^{2}}{4 a}\right), \quad a \neq 0, v=\infty, 2,3,5, \cdots,
$$

where index $v$ denotes real $(v=\infty)$ and $p$-adic cases, $\chi_{v}$ is an additive character: $\chi_{\infty}(x)=\exp (-2 \pi i x), \chi_{p}(x)=\exp \left(2 \pi i\{x\}_{p}\right)$, where $\{x\}_{p}$ is the fractional part of $x \in \mathbb{Q}_{p}$, and $\lambda_{v}(a)$ is the complex-valued arithmetic function. ${ }^{8}$ An adele ${ }^{21}$ is an infinite sequence $a=\left(a_{\infty}, a_{2}, \ldots, a_{p}, \ldots\right)$, where $a_{\infty} \in \mathbb{R} \equiv \mathbb{Q}_{\infty}, a_{p} \in \mathbb{Q}_{p}$ with a restriction that $a_{p} \in \mathbb{Z}_{p}$ for all but a finite set $S$ of primes $p$. The set of all adeles $\mathbb{A}$ may be regarded as a subset of direct topological product $\mathbb{Q}_{\infty} \times \prod_{p} \mathbb{Q}_{p}$. $\mathbb{A}$ is a topological space, and can be considered as a ring with respect to the componentwise addition and multiplication. An elementary function on adelic ring $\mathbb{A}$ is

$$
\varphi(x)=\varphi_{\infty}\left(x_{\infty}\right) \prod_{p} \varphi_{p}\left(x_{p}\right)=\prod_{v} \varphi_{v}\left(x_{v}\right)
$$


with the main restriction that $\varphi(x)$ must satisfy $\varphi_{p}\left(x_{p}\right)=\Omega\left(\left|x_{p}\right|_{p}\right)$ for all but a finite number of $p$. Characteristic function on $p$-adic integers $\mathbb{Z}_{p}$ is defined by $\Omega\left(|x|_{p}\right)=1,0 \leq|x|_{p} \leq 1$ and $\Omega\left(|x|_{p}\right)=0,|x|_{p}>1$.

The Fourier transform of the characteristic function (vacuum state) $\Omega\left(\left|x_{p}\right|\right.$ ) is $\Omega\left(\left|k_{p}\right|\right)$. All finite linear combinations of elementary functions (5) make the set $\mathcal{D}(\mathbb{A})$ of the Schwartz-Bruhat functions. The Hilbert space $L_{2}(\mathbb{A})$ is a space of complexvalued functions $\psi_{1}(x), \psi_{2}(x), \ldots$, with the scalar product and norm.

In foundations of standard QM one usually starts with a representation of the canonical commutation relation (2). In formulation of $p$-adic QM ${ }^{11}$ the multiplication $\hat{q} \psi \rightarrow x \psi$ has no meaning for $x \in \mathbb{Q}_{p}$ and $\psi(x) \in \mathbb{C}$. In the real case momentum and hamiltonian are infinitesimal generators of space and time translations, but, since $\mathbb{Q}_{p}$ is totally disconnected field, these infinitesimal transformations become meaningless. However, finite transformations remain meaningful and the corresponding Weyl and evolution operators are $p$-adically well defined.

Canonical commutation relation (2) in $p$-adic case can be represented by the Weyl operators $(h=1)$

$$
\begin{gathered}
\hat{Q}_{p}(\alpha) \psi_{p}(x)=\chi_{p}(\alpha x) \psi_{p}(x), \\
\hat{K}_{p}(\beta) \psi(x)=\psi_{p}(x+\beta), \\
\hat{Q}_{p}(\alpha) \hat{K}_{p}(\beta)=\chi_{p}(\alpha \beta) \hat{K}_{p}(\beta) \hat{Q}_{p}(\alpha) .
\end{gathered}
$$

It is possible to introduce the family of unitary operators

$$
\hat{W}_{p}(z)=\chi_{p}\left(-\frac{1}{2} q k\right) \hat{K}_{p}(\beta) \hat{Q}_{p}(\alpha), \quad z \in \mathbb{Q}_{p} \times \mathbb{Q}_{p},
$$

that is a unitary representation of the Heisenberg-Weyl group.

The dynamics of a $p$-adic quantum model is described by a unitary operator of evolution $U(t)$ formulated in terms of its kernel $\mathcal{K}_{t}(x, y)$. In this way ${ }^{11} p$-adic QM is given by a triple $\left(L_{2}\left(\mathbb{Q}_{p}\right), W_{p}\left(z_{p}\right), U_{p}\left(t_{p}\right)\right)$.

Keeping in mind that standard QM can be also given as the corresponding triple, ordinary and $p$-adic QM can be unified in the form of adelic QM 12

$$
\left(L_{2}(A), W(z), U(t)\right),
$$

$L_{2}(\mathbb{A})$ is the Hilbert space on $\mathbb{A}, W(z)$ is a unitary representation of the HeisenbergWeyl group on $L_{2}(\mathbb{A})$ and $U(t)$ is a unitary representation of the evolution operator on $L_{2}(\mathbb{A})$. The evolution operator $U(t)$ is defined by

$$
U(t) \psi(x)=\int_{\mathbb{A}} \mathcal{K}_{t}(x, y) \psi(y) d y=\prod_{v} \int_{\mathbb{Q}_{v}} \mathcal{K}_{t}^{(v)}\left(x_{v}, y_{v}\right) \psi^{(v)}\left(y_{v}\right) d y_{v} .
$$

Note that any adelic eigenfunction has the form

$$
\Psi(x)=\Psi_{\infty}\left(x_{\infty}\right) \prod_{p \in S} \Psi_{p}\left(x_{p}\right) \prod_{p \notin S} \Omega\left(\left|x_{p}\right|_{p}\right), \quad x \in \mathbb{A},
$$


where $\Psi_{\infty} \in L_{2}(\mathbb{R}), \Psi_{p} \in L_{2}\left(\mathbb{Q}_{p}\right)$. In the low-energy limit adelic QM becomes ordinary one.

A suitable way to calculate propagator in $p$-adic QM is by $p$-adic generalization of Feynman's path integral.11 There is no natural ordering on $\mathbb{Q}_{p}$. However, a bijective continuous map $\varphi$ from the set of $p$-adic numbers $\mathbb{Q}_{p}$ to the subset $\varphi\left(\mathbb{Q}_{p}\right)$

of real numbers $\mathbb{R}$ was proposed ${ }^{8}$ This map can be defined by (for an older injective version see Ref. 18)

$$
\varphi(x)=|x|_{p} \sum_{k=0}^{\infty} x_{k} p^{-2 k} .
$$

Then, a linear order on $\mathbb{Q}_{p}$ is given by the following definition: $x<y$ if $|x|_{p}<|y|_{p}$ or when $|x|_{p}=|y|_{p}$ there exists such index $m \geq 0$ that digits satisfy $x_{0}=y_{0}, x_{1}=$ $y_{1}, \cdots, x_{m-1}=y_{m-1}, x_{m}<y_{m}$. One can say: $\varphi(x)>\varphi(y)$ iff $x>y$.

In the case of harmonic oscillator $\frac{18}{18}$ it was shown that there exists the limit

$$
\begin{aligned}
& \mathcal{K}_{p}\left(x^{\prime \prime}, t^{\prime \prime} ; x^{\prime}, t^{\prime}\right)=\lim _{n \rightarrow \infty} \mathcal{K}_{p}^{(n)}\left(x^{\prime \prime}, t^{\prime \prime} ; x^{\prime}, t^{\prime}\right)=\lim _{n \rightarrow \infty} N_{p}^{(n)}\left(t^{\prime \prime}, t^{\prime}\right) \\
& \times \int_{\mathbb{Q}_{p}} \cdots \int_{\mathbb{Q}_{p}} \chi_{p}\left(-\frac{1}{h} \sum_{i=1}^{n} \bar{S}\left(q_{i}, t_{i} ; q_{i-1}, t_{i-1}\right)\right) d q_{1} \cdots d q_{n-1},
\end{aligned}
$$

where $N_{p}^{(n)}\left(t^{\prime \prime}, t^{\prime}\right)$ is the corresponding normalization factor for the harmonic oscillator. The subdivision of $p$-adic time segment $t_{0}<t_{1}<\cdots<t_{n-1}<t_{n}$ is made according to linear order on $\mathbb{Q}_{p}$. In a similar way we have calculated path integrals for a few quantum models. Moreover, we were able to obtain general expression for the propagator of the systems with quadratic action (for the details see Ref. 22), without ordering

$$
\mathcal{K}_{p}\left(x^{\prime \prime}, t^{\prime \prime} ; x^{\prime}, t^{\prime}\right)=\lambda_{p}\left(-\frac{1}{2 h} \frac{\partial^{2} \bar{S}}{\partial x^{\prime \prime} \partial x^{\prime}}\right)\left|\frac{1}{h} \frac{\partial^{2} \bar{S}}{\partial x^{\prime \prime} \partial x^{\prime}}\right|_{p}^{\frac{1}{2}} \chi_{p}\left(-\frac{1}{h} \bar{S}\left(x^{\prime \prime}, t^{\prime \prime} ; x^{\prime}, t^{\prime}\right)\right) .
$$

Replacing an index $p$ with $v$ in (15) we can write quantum-mechanical amplitude $\mathcal{K}$ in ordinary and all $p$-adic cases in the same, compact (and adelic) form.

\section{Relations Between Noncommutative and $p$-Adic QM}

Noncommutative geometry is geometry which is described by an associative algebra $\mathcal{A}$, which is usually noncommutative and in which the set of points, if it exists at all, is relegated to a secondary role. Noncommutative spaces have arisen in investigation of brane configurations in string and M-theory. Since the one-particle sector of field theories leads to QM, a study of this topic has attracted much interest. For single particle QM, the corresponding Heisenberg algebra is needed. In addition to (1) and (2) one chooses

$$
\left[p^{i}, p^{j}\right]=i \Phi^{i j} .
$$

There are a lot of possibilities in choosing $\theta$ and $\Phi ! 5]$ Although one can take $\theta^{i j}$ and $\Phi^{i j}$ to be antisymmetric nonconstant tensors (matrices), often the simplest 
nontrivial case is considered: $\theta^{i j}=$ const and $\Phi^{i j}=0$. Another realization of noncommutativity is possible by $q$-deformation of a space, for example, Manin plane $x y=q y x$ and $q$-deformed "classical" phase space $p x=q p x$. This approach leads to a latticelike (discrete) structure of space-time.23

A field $\Psi(x)$ as a function of the noncommuting coordinates $x$ can be used as Schrödinger wave function obeying the free field equation. Other realization, based on star product $(v * \Psi)$ instead of standard multiplication $(v \cdot \Psi)$ of a potential and wave function have been considered in corresponding Schrödinger equation as well (i.e. see Ref. 24).

The passage from one level of physical theory to a more refined one, using what mathematicians call deformation theory, is nothing extraordinary new. In a similar way, there is an old idea that QM is some kind of deformed classical mechanics. For a review see Ref. 25, In fact, deformation quantization is closely related to Weyl quantization, briefly sketched out in the previous section.

One direction of the investigation led to Moyal bracket and Moyal (star) product $\frac{16}{16}$ widely used now in noncommutative QM

$$
f *_{m} g=\chi_{\infty}\left(-\frac{h}{8 \pi^{2}} P\right)(f, g)=f g+\sum_{r=1}^{\infty}\left(\frac{i h}{4 \pi}\right)^{r} P^{r}(f, g) .
$$

Several integral formulas have been introduced for the star product and a (formal) parameter of deformation is finally related to some form of Planck constant $h$. Quantization can be taken as a deformation of the standard associative and commutative product, now called a star product, of classical observables driven by the Poisson bracket $P$. By the intuition, classical mechanics is understood as the limit of QM when $h \rightarrow 0$.

Some connections between $p$-adic analysis and quantum deformations has been noticed. It has been observed that the Haar measure on $S U_{q}(2)$ coincides with the Haar measure on the field of $p$-adic numbers $\mathbb{Q}_{p}$ if $q=1 / p ![6$ There is a potential such that the spectrum of the $p$-adic Schrödinger-like (diffusion) equation 8

$$
D \psi(x)+V\left(|x|_{p}\right) \psi(x)=E \psi(x)
$$

is the same as in the case of $q$-deformed oscillator found by Biedenharn 26 for $q=$ $1 / p$. For more details see Ref. 6 .

In a development of the representation theory for the star product algebras in deformation quantization some non-Archimedean behavior is noted 16 . We find this relation very intriguing and discuss it in more details. Recall that a star product $*$, on a Poisson manifold $(\mathrm{M}, \pi)$ is a (formal) associative $\mathbb{C}[[\lambda]]$ - bilinear product for $C^{\infty}(M)[[\lambda]]$

$$
f * g=\sum_{r=0}^{\infty} \lambda^{r} C_{r}(f, g),
$$

with bidifferential operators $C_{r} . \mathbb{C}[[\lambda]]$ is an algebra of formal (in a formal parameter $\lambda$ ) series $\sum_{r=0}^{\infty} \lambda^{r} C_{r}, C_{r} \in \mathbb{C}$ (a convergence of this series is still not un- 
der a consideration). $C^{\infty}(M)[[\lambda]]$ is the space of formal series of smooth functions $\left(x \in M, f_{r}(x) \in C^{\infty}(M)\right)$, for a fixed but variable $x$. With interpretation $\lambda \leftrightarrow \hbar$ we identify $C^{\infty}(M)[[\lambda]]$ with the algebra of observables of the quantum system corresponding to $(M, \pi)$. Let $R$ be on an ordered (commutative associative unital) ring $R{ }^{16}$ Let us note that a concept of ordered ring is necessary if one wishes to define the positive functionals on a $C^{*}$ algebra $(C=R(i)=\{a+b i, a, b \in R\})$. It is related to Gelfand-Naimark's theorem on commutative spaces. By means of the positive functionals on $C^{*}$ algebras we can reconstruct the (points on) "starting" manifold, the one on which an algebra of complex function will give the "original" $C^{*}$ algebra. This is a motivation for generalization on noncommutative space. In this case, the corresponding product of formal functions will be the $*$ product. Now, $R[[\lambda]]$ will be an algebra of formal series on the ordered ring $R$. Then, if $R$ is an ordered ring, $R[[\lambda]]$ will be ordered in a canonical way, too, by the definition

$$
\sum_{r=r_{0}}^{\infty} \lambda^{r} a_{r}>0, \text { if } a_{r_{0}}>0 .
$$

In other words, a formal series in $R[[\lambda]]$ will be a "positive" one if first nonzero coefficient (an element of ordered ring $R$ is positive). It should be noted that the concept of ordered ring fits naturally with formal power series and thus to Gerstenhaber's deformation theory 27 Then $R[[\lambda]]$ will be non-Archimedean! For example, if we take that $a_{0}=-1$, and $a_{1}=n(n \in \mathbb{N}$, because for any commutative ordered ring with unit, set of integers is embedded in $R, \mathbb{N} \subset R$ ), all other coefficients can be zero, we have $-1+n \lambda<0$ or $n \lambda<1$ for all $n \in \mathbb{Z}$. The interpretation in formal theory is that the deformation parameter $\lambda$ is "very small" compared to the other numbers in R. We see that Waldmann's definition of the ordered algebra of formal series on ordered ring $R$ immediately leads to the non-archimedean "structure".

This result is a good indication to use ultrametric spaces and $p$-adics when physical deformation parameter is very small. We would like to underline that Zelenov's ordering by means of map (13) $\mathbb{Q}_{p}$, i.e. on normed, ultrametric algebra in frame of $p$-adic QM for $\lambda=1 / p^{2}$ is related to the ordering of formal series on ordered rings in the frame of deformation quantization! This is a quite intriguing coincidence of a strong potential for further investigation.

\section{The Adelic Moyal Product and Noncommutative QM}

The presented connections between noncommutative vs. "nonarchimedean" QM suggest a need to formulate a quantum theory that may connect as much as possible nonarchimedean and noncommutative effects and structures. At the present level of quantum theory on adeles, a formulation of noncommutative adelic QM seems to be the most promising attempt.

A simple enough frame for that might be the representation of an algebra of operators (11), (2) and (16). It could be done by linear transformations on the corresponding simplectic structure and deformed and extended bilinear product $B$. 
Correspondence between classical functions and quantum operators would be provided by Weyl quantisation. An equivalent formulation of noncommutative adelic QM by the triple $\left(L_{2}\left(A_{\theta}\right), W_{\theta}(z), U_{\theta}(t)\right)$, does not seem to have principles obstacles. In this approach an adele of coordinates $x_{A}$ would be replaced by a series of noncommutative operators $\hat{x}_{A}$, where adelic properties of corresponding eigenvalues are still "preserved".

Now, we have to consider a $p$-adic and adelic generalization of the Moyal product. Let us consider classical space with coordinates $x^{1}, x^{2}, \cdots, x^{D}$. Let $f(x)$ be a classical function $f(x)=f\left(x^{1}, x^{2}, \cdots, x^{D}\right)$. Then, with respect to the Fourier transformations and the usual Weyl quantization, we have

$$
\hat{f}(x)=\int_{\mathbb{Q}_{\infty}^{D}} d k \chi_{\infty}(-k \hat{x}) \tilde{f}(k) \equiv f(\hat{x}) .
$$

Let us now have two classical functions $f(x)$ and $g(x)$ and we are interested in operator product $\hat{f}(x) \hat{g}(x)$. In the real case this operator product is

$$
(\hat{f} \cdot \hat{g})(x)=\iint d k d k^{\prime} \chi_{\infty}(-k \hat{x}) \chi_{\infty}\left(-k^{\prime} \hat{x}\right) \tilde{f}(k) \tilde{g}\left(k^{\prime}\right) .
$$

Using the Baker-Campbell-Hausdorff formula, the relation (11) and then the coordinate representation one finds the Moyal product in the form

$$
(f * g)(x)=\int_{\mathbb{Q}_{p}^{D}} \int_{\mathbb{Q}_{p}^{D}} d k d k^{\prime} \chi_{v}\left(-\left(k+k^{\prime}\right) x+\frac{1}{2} k_{i} k_{j}^{\prime} \theta^{i j}\right) \tilde{f}(k) \tilde{g}\left(k^{\prime}\right) .
$$

Note that we already used our generalization from $\mathbb{Q}_{\infty}$ to $\mathbb{Q}_{v}$. In the real case we use $k_{i} \rightarrow-(i / 2 \pi)\left(\partial / \partial x^{i}\right)$ and obtain the well known form $(f * g)(x)=$ $\left.\chi_{\infty}\left(-\frac{\theta^{i j}}{2(2 \pi)^{2}} \frac{\partial}{\partial y^{i}} \frac{\partial}{\partial z^{j}}\right) f(y) g(z)\right|_{y=z=x}$. In the $p$-adic case such a straightforward generalization is not possible ${ }^{19 \mid 28}$ (but some kind of pseudodifferentiation could be useful). Thus, as the $p$-adic Moyal product we take

$$
(f * g)(x)=\int_{\mathbb{Q}_{p}^{D}} \int_{\mathbb{Q}_{p}^{D}} d k d k^{\prime} \chi_{p}\left(-\left(x^{i} k_{i}+x^{j} k_{j}^{\prime}\right)+\frac{1}{2} k_{i} k_{j}^{\prime} \theta^{i j}\right) \tilde{f}(k) \tilde{g}\left(k^{\prime}\right) .
$$

We can write down the adelic Moyal product of "classical" adelic functions $f_{A}=$ $\left(f_{\infty}, f_{2}, \ldots, f_{p}, \ldots\right), g_{A}=\left(g_{\infty}, g_{2}, \ldots, g_{p}, \ldots\right)$ on $\mathbb{R} \times \prod_{p \in S} \mathbb{Q}_{p} \times \prod_{p \notin S} \mathbb{Z}_{p}$ space

$$
(f * g)(x)=\int_{\mathbb{A}^{D}} \int_{\mathbb{A}^{D}} d k d k^{\prime} \chi\left(-\left(x^{i} k_{i}+x^{j} k_{j}^{\prime}\right)+\frac{1}{2} k_{i} k_{j}^{\prime} \theta^{i j}\right) \tilde{f}_{A}(k) \tilde{g}_{A}\left(k^{\prime}\right) .
$$

Taking into account (5), (22) and the property of the Fourier transform of $\Omega$ function, one has

$$
\begin{array}{r}
(f * g)(x)=\left.\chi_{\infty}\left(-\frac{\theta^{i j}}{2(2 \pi)^{2}} \frac{\partial}{\partial y^{i}} \frac{\partial}{\partial z^{j}}\right) f(y) g(z)\right|_{y=z=x} \\
\times \prod_{p \in S} \int_{\mathbb{Q}_{p}^{D}} \int_{\mathbb{Q}_{p}^{D}} d k d k^{\prime} \chi_{p}\left(-\left(x^{i} k_{i}+x^{j} k_{j}^{\prime}\right)+\frac{1}{2} k_{i} k_{j}^{\prime} \theta^{i j}\right) \tilde{f}_{p}(k) \tilde{g}_{p}\left(k^{\prime}\right) \\
\times \prod_{p \notin S} \int_{\mathbb{Z}_{p}^{D}} \int_{\mathbb{Z}_{p}^{D}} d k d k^{\prime} \chi_{p}\left(-\left(x^{i} k_{i}+x^{j} k_{j}^{\prime}\right)+\frac{1}{2} k_{i} k_{j}^{\prime} \theta^{i j}\right) .
\end{array}
$$


It can be shown that if for all $p, \varphi(x)=\Omega(x)$, the adelic Moyal product becomes real one, with some condition imposed on $\theta$.

\section{Noncommutativity in Quantum Cosmology}

The words "quantum" and "cosmology" do appear to some physicists to be inherently incompatible. We usually think of cosmology in terms of the very large structure of the universe and of quantum phenomena in terms of the very small. However, since gravity is the dominating interaction on cosmic scales, a quantum theory of gravity is needed as a formal prerequisite for quantum cosmology. Most work in quantum cosmology is based on the Wheeler-DeWitt equation of quantum geometrodynamics. In quantum mechanics and quantum field theory, path integrals provide a convenient tool for a wide range of applications. In quantum gravity, a path-integral formulation would have to employ a sum over all four-metrics for a given topology.

In this paper we consider $(4+\mathrm{D})$ Kaluza-Klein models, or more precisely a particular, simpler model, the so called $(4+1)$ "empty" model.

We start with the metric considered in Refs. 29, 30, 31, 32, in which spacetime is of a Friedman-Robertson-Walker type, having a compactified space which is assumed to be the circle $S^{1}$. We adopt the chart $\left\{t, r^{i}, \rho\right\}$ with $t, r^{i}$ and $\rho$ denoting the time, the space coordinates and the compactified space coordinate, respectively

$$
d s^{2}=-N^{2} d t^{2}+R^{2}(t) \frac{d r^{i} d r^{i}}{\left(1+\frac{\kappa r^{2}}{4}\right)^{2}}+a^{2} d \rho^{2},
$$

where $\kappa=0, \pm 1$ and $R(t), a(t)$ are the scale factors of the universe and compact

dimension, respectively. The integration of the Einstein-Hilbert action for such an empty $(4+1)$ dimensional Kaluza-Klein universe with cosmological constant $\Lambda$

$$
S=\int \sqrt{-g}(\tilde{R}-\Lambda) d t d^{3} r d \rho,
$$

( $\tilde{R}$ is a curvature scalar corresponding to metric (27)) over spatial dimensions gives an effective Lagrangian in the minisuperspace $(R, a)$

$$
L=\frac{1}{2 N} R a \dot{R}^{2}+\frac{1}{2 N} R^{2} \dot{R} \dot{a}-\frac{1}{2} N \kappa R a+\frac{1}{6} N \Lambda R^{3} a .
$$

\subsection{Commutative model over real space}

By defining $\omega^{2}=-\frac{2 \Lambda}{3}(\Lambda<0)$ and changing variables as

$$
u=\frac{1}{\sqrt{8}}\left[R^{2}+R a-\frac{3 \kappa}{\Lambda}\right], \quad v=\frac{1}{\sqrt{8}}\left[R^{2}-R a-\frac{3 \kappa}{\Lambda}\right],
$$

in the "new" minisuperspace $(u, v)$, Lagrangian takes the form

$$
L=\frac{1}{2 N}\left(\dot{u}^{2}-N^{2} \omega^{2} u^{2}\right)-\frac{1}{2 N}\left(\dot{v}^{2}-N^{2} \omega^{2} v^{2}\right),
$$


which describes an isotropic oscillator-ghost-oscillator system. Corresponding equations of motion and solutions are

$$
\begin{gathered}
\ddot{u}+N^{2} \omega^{2} u=0, \quad \ddot{v}+N^{2} \omega^{2} v=0, \\
u(t)=A \cos N \omega t+B \sin N \omega t, \quad v(t)=C \cos N \omega t+D \sin N \omega t .
\end{gathered}
$$

Classical action is

$$
\begin{gathered}
\bar{S}\left(u^{\prime \prime}, v^{\prime \prime}, N ; u^{\prime}, v^{\prime}, 0\right) \\
=\frac{1}{2} \omega\left[\left(u^{\prime \prime 2}+u^{\prime 2}-v^{\prime \prime 2}-v^{\prime 2}\right) \cot N \omega+\left(v^{\prime} v^{\prime \prime}-u^{\prime} u^{\prime \prime}\right) \frac{2}{\sin N \omega}\right],
\end{gathered}
$$

while the propagator is

$$
\mathcal{K}\left(u^{\prime \prime}, v^{\prime \prime}, N ; u^{\prime}, v^{\prime}, 0\right)=\frac{1}{i} \sqrt{\frac{\omega^{2}}{\sin N \omega}} \times \exp \left(2 \pi i \bar{S}\left(u^{\prime \prime}, v^{\prime \prime}, N ; u^{\prime}, v^{\prime}, 0\right)\right) .
$$

To obtain the energy eigenstates and eigen vectors, we need to recast the propagator (35) in a form that permits a direct comparison with the spectral representation for the Feynman propagator given by

$$
\mathcal{K}\left(u^{\prime \prime}, v^{\prime \prime}, N ; u^{\prime}, v^{\prime}\right)=\Theta(N) \sum_{l} \Phi_{l}^{\left(m_{1}, m_{2}\right)}\left(u^{\prime \prime}, v^{\prime \prime}\right) \Phi_{l}^{*\left(m_{1}, m_{2}\right)}\left(u^{\prime}, v^{\prime}\right) e^{-i \frac{N E_{n, m}}{\hbar}} .
$$

Corresponding Wheeler-Dewitt equation for this model in the minisuperspace $(u, v)$ is $(N=1)$

$$
\left(\frac{\partial^{2}}{\partial u^{2}}-\frac{\partial^{2}}{\partial v^{2}}-\omega^{2} u^{2}+\omega^{2} v^{2}\right) \Psi(u, v)=0 .
$$

It has oscillator-ghost-oscillator solutions belonging to the Hilbert space $\mathcal{H}^{\left(m_{1}, m_{2}\right)}\left(\mathcal{L}^{2}\right)$ as

$$
\Psi^{\left(m_{1}, m_{2}\right)}(u, v)=\sum_{l=0}^{\infty} c_{l} \Phi_{l}^{\left(m_{1}, m_{2}\right)}(u, v)
$$

with $m_{1}, m_{2} \geq 0$ and $c_{l} \in C$. For more details about basis solutions $\Phi_{l}^{\left(m_{1}, m_{2}\right)}(u, v)$ see Ref. [30,

\subsection{Commutative model over p-adic space}

Let us consider this model on a $p$-adic space. The main relations (27) and (28) connected with the $(4+1)$-dimensional model in the $p$-adic case are the same as in the real case. Now, the effective $p$-adic valued Lagrangian in the minisuperspace $(R, a)$ is

$$
L=\frac{1}{2 N} R a \dot{R}^{2}+\frac{1}{2 N} R^{2} \dot{R} \dot{a}-\frac{1}{2} N \kappa R a+\frac{1}{6} N \Lambda R^{3} a .
$$


Again, by defining $\omega^{2}=-\frac{2 \Lambda}{3}(\Lambda<0)$ and changing variables as

$$
u=\frac{1}{\sqrt{8}}\left[R^{2}+R a-\frac{3 \kappa}{\Lambda}\right], \quad v=\frac{1}{\sqrt{8}}\left[R^{2}-R a-\frac{3 \kappa}{\Lambda}\right],
$$

in the "new" minisuperspace $(u, v)$, Lagrangian takes the form

$$
L=\frac{1}{2 N}\left(\dot{u}^{2}-N^{2} \omega^{2} u^{2}\right)-\frac{1}{2 N}\left(\dot{v}^{2}-N^{2} \omega^{2} v^{2}\right) .
$$

Corresponding equations of motion has formally the same form as (32), but it's solutions have a different domain of convergence $8 \mid 32$

$$
u(t)=A \cos N \omega t+B \sin N \omega t, \quad v(t)=C \cos N \omega t+D \sin N \omega t .
$$

Classical action is

$$
\begin{gathered}
\bar{S}_{p}\left(u^{\prime \prime}, v^{\prime \prime}, N ; u^{\prime}, v^{\prime}, 0\right) \\
=\frac{1}{2} \omega\left[\left(u^{\prime \prime 2}+u^{\prime 2}-v^{\prime \prime 2}-v^{\prime 2}\right) \cot N \omega+\left(v^{\prime} v^{\prime \prime}-u^{\prime} u^{\prime \prime}\right) \frac{2}{\sin N \omega}\right] .
\end{gathered}
$$

This action leads to the propagator 33

$$
\mathcal{K}_{p}\left(v^{\prime \prime}, u^{\prime \prime}, N ; v^{\prime}, u^{\prime}, 0\right)=\frac{1}{|N|_{p}} \chi_{p}\left(\frac{\omega\left(u^{\prime \prime 2}+u^{\prime 2}-v^{\prime 2}-v^{\prime \prime 2}\right)}{2 \tan N \omega}+\frac{\omega\left(v^{\prime} v^{\prime \prime}-u^{\prime} u^{\prime \prime}\right)}{\sin N \omega}\right) .
$$

In the $p$-adic region of convergence of analytic functions $\sin x$ and $\tan x$, which is $G_{p}=\left\{x \in Q_{p}:|x|_{p} \leq|2 p|_{p}\right\}$, exist vacuum states $\Omega\left(|u|_{p}\right) \Omega\left(|v|_{p}\right), \Omega\left(p^{\nu}|u|_{p}\right)$ $\times \Omega\left(p^{\mu}|v|_{p}\right), \nu, \mu=1,2,3$, and also

$$
\Psi_{p}(x, y)=\left\{\begin{array}{l}
\delta\left(p^{\nu}-|x|_{p}\right) \delta\left(p^{\mu}-|y|_{p}\right),|N|_{p} \leq p^{2 \nu-2},|N|_{p} \leq p^{2 \mu-2} \\
\delta\left(2^{\nu}-|x|_{2}\right) \delta\left(2^{\mu}-|y|_{2}\right),|N|_{2} \leq 2^{2 \nu-3},|N|_{2} \leq 2^{2 \mu-3}
\end{array}\right.
$$

where $\mu, \nu=0,-1,-2, \cdots$.

Some $4(=3+1)$-dimensional quantum cosmological models, which in $p$-adic sector looks like two decoupled harmonic oscillators, were analyzed in details in Refs. 13, 19, 28, 32,

\subsection{Noncommutative case}

In the previous section we assumed that in $(u, v)$ minisuperspace held

$$
[u, v]=0, \quad\left[u, p_{u}\right]=\left[v, p_{v}\right]=i \hbar, \quad\left[p_{u}, p_{v}\right]=0,
$$

(generalized momenta are $p_{u}=\frac{\dot{u}}{N}$ and $p_{v}=\frac{\dot{v}}{N}$ ). In the noncommutative case we deal with the same Lagrangian, but with algebra

$$
[u, v]=i \theta, \quad\left[u, p_{u}\right]=\left[v, p_{v}\right]=i \hbar, \quad\left[p_{u}, p_{v}\right]=0 .
$$


By the transformation

$$
u=u-\frac{\theta}{2} p_{v}, \quad v=v+\frac{\theta}{2} p_{u},
$$

we can represent this model as a commuting but with the Lagrangian

$$
L_{\theta}=\frac{\omega^{2}}{\omega_{\theta}^{2}}\left[\frac{1}{2 N}\left(\dot{u}^{2}-N^{2} \omega_{\theta}^{2} u^{2}\right)-\frac{1}{2 N}\left(\dot{v}^{2}-N^{2} \omega_{\theta}^{2} v^{2}\right)+\frac{1}{2 N} \omega_{\theta}^{2} \theta(u \dot{v}+\dot{u} v)\right],
$$

where $\omega_{\theta}^{2}=\frac{\omega^{2}}{1+\frac{\omega^{2} \theta^{2}}{4}}$. The equations of motion and solutions are

$$
\begin{gathered}
\ddot{u}+N^{2} \omega_{\theta}^{2} u=0, \quad \ddot{v}+N^{2} \omega_{\theta}^{2} v=0, \\
u(t)=A \cos N \omega_{\theta} t+B \sin N \omega_{\theta} t, \quad v(t)=C \cos N \omega_{\theta} t+D \sin N \omega_{\theta} t .
\end{gathered}
$$

From the classical action

$$
\begin{gathered}
\bar{S}_{\theta}\left(u^{\prime \prime}, v^{\prime \prime}, N ; u^{\prime}, v^{\prime}, 0\right)=\frac{1}{2} \omega \sqrt{1+\frac{\omega^{2} \theta^{2}}{4}} \\
\times\left[\left(u^{\prime \prime 2}+u^{\prime 2}-v^{\prime \prime 2}-v^{\prime 2}\right) \cot N \omega_{\theta}-\left(u^{\prime} u^{\prime \prime}-v^{\prime} v^{\prime \prime}\right) \frac{2}{\sin N \omega_{\theta}}+\frac{\theta \omega_{\theta}}{N}\left(u^{\prime \prime} v^{\prime \prime}-u^{\prime} v^{\prime}\right)\right],
\end{gathered}
$$

we first get

$$
\left|\begin{array}{l}
-\frac{\partial^{2} \bar{S}}{\partial u^{\prime} u^{\prime \prime}}-\frac{\partial^{2} \bar{S}}{\partial u^{\prime} v^{\prime \prime}} \\
-\frac{\partial^{2} \bar{S}}{\partial v^{\prime} \partial u^{\prime \prime}}-\frac{\partial^{2} \bar{S}}{\partial v^{\prime} \partial v^{\prime \prime}}
\end{array}\right|^{1 / 2}=\sqrt{1+\frac{\omega^{2} \theta^{2}}{4}} \frac{\omega}{\left|\sin N \omega_{\theta}\right|},
$$

and finally

$$
\begin{aligned}
& \mathcal{K}_{\theta}\left(u^{\prime \prime}, v^{\prime \prime}, N ; u^{\prime}, v^{\prime}, 0\right)=\frac{1}{i} \sqrt{1+\frac{\omega^{2} \theta^{2}}{4}} \sqrt{\frac{\omega^{2}}{\sin N \omega_{\theta}}} \\
& \times \exp \left(2 \pi i \bar{S}_{\theta}\left(u^{\prime \prime}, v^{\prime \prime}, N ; u^{\prime}, v^{\prime}, 0\right)\right) .
\end{aligned}
$$

In commutative regime (obtained for the $\theta=0$ ) we have 20

$$
\mathcal{K}\left(u^{\prime \prime}, v^{\prime \prime}, N ; u^{\prime}, v^{\prime}, 0\right)=\frac{1}{i} \sqrt{\frac{\omega^{2}}{\sin N \omega}} \times \exp \left(2 \pi i \bar{S}\left(u^{\prime \prime}, v^{\prime \prime}, N ; u^{\prime}, v^{\prime}, 0\right)\right) .
$$

This example and remarkable result shows that exploring connections between standard, nonarchimedean and noncommutative theory, including quantum cosmology, are very interesting. Applications of noncommutativity in cosmology remain current at present, $34 \mid 35$ with our belief that noncommutative and nonlocal tachyonic models of inflation deserve more attention in future. 


\section{Acknowledgments}

Work on this paper is partially supported by the ICTP - SEENET-MTP grant PRJ-09 within the framework of the SEENET-MTP Network and by the Ministry of Education and Science Republic of Serbia under Grant 176021. A part of the whole research program was cofinanced by the DFG grant "Noncommutative space-time structure - Cooperation with Balkan Countries" and the DAAD project fellow "Nonarchimedean Analysis, $p$-Adic Quantum Mechanics and Quantum Deformation". I would like to thank P. Aschieri, I. Baković, B. Dragović, B. Jurco, L. Möller, Lj. Nešić and S. Waldmann for many useful discussions on topics considered in this paper. My honest thankfulness to J. Dimitrijević-Savić for her continual help in proofreading of many of my and SEENET-MTP publications. A personal gratitude to my younger collaborators and colleagues D. Dimitrijević, M. Milošević and J. Stanković for their technical help in preparation of the manuscript and their outstanding service in the SEENET-MTP Network mission.

\section{References}

1. L. Möller, Julius Wess - a Scientist in Global Responsibility, in this volume.

2. http://www.seenet-mtp.info/statement-of-intention.

3. Mathematical, Theoretical and Phenomenological Ccallenges Beyond the Standard Model, eds. G. S. Djordjevic, Lj. Nesic and J. Wess (World Scientific, Singapore, 2005), pp. 1-280.

4. http://tesla.pmf.ni.ac.rs/f_odeljenje/.

5. W. Behr, N. G. Desphande, G. Duplancic, P. Shupp, J. Trampetic and J. Wess, Eur. Phys. J. C 29, 441 (2003).

6. I. Ya. Aref'eva and I. V. Volovich, Phys. Lett. B 268, 179 (1991).

7. I. V. Volovich, Class. Quantum Grav. 4, L83 (1987).

8. V. S. Vladimirov, I. V. Volovich and E. I. Zelenov, p-Adic Analysis and Mathematical Physics, (World Scientific, Singapore, 1994).

9. L. Brekke and P. G. O. Freund, Phys. Rep. 233, 1 (1993).

10. B. Dragovich, A. Yu Khrennikov, S. V. Kozyrev and I. V. Volovich, Anal. Appl 1, 1 (2009).

11. V. S. Vladimirov and I. V. Volovich, Comm. Math. Phys. 123, 659 (1989).

12. B. Dragovich, Int. J. Mod. Phys. A 10, 2349 (1995).

13. G. S. Djordjevic, B. Dragovich, Lj. Nesic and I. V. Volovich, Int. J. Mod. Phys. A 17, 1413 (2002).

14. D. Dimitrijevic, G. S. Djordjevic and Lj. Nesic, Nonarchimedean and Noncommutative Quantum Cosmology and Tachyons, in BW2007 WORKSHOP, III Southeastern European Workshop Challenges Beyond the Standard Model, eds. G. S. Djordjevic, Lj. Nesic and M. Haack (2007), p. 13.

15. R. Jackiw, Nucl. Phys. Proc. Suppl. 108, 30 (2002).

16. S. Waldmann: On the Representation Theory of Deformation Quantization, math.QA/0107112

17. D. D. Dimitrijevic, G. S. Djordjevic and S. Waldmann, Ultrametric Generalization of the Moyal Product, in Proc. XVI International Conference of Applied Mathematics, Serbia and Montenegro, eds. D. Herceg, K. Surla and N. Krejic, Novi Sad (2004), p. 16.

18. E. I. Zelenov, J. Math. Phys. 32, 147 (1991). 
19. G. Djordjevic, B. Dragovich and Lj. Nesic, Adelic Quantum Mechanics: Nonarchimedean and Noncommuative Aspects, in Proc. NATO ARW Noncommutative Structures in Mathematics and Physics, eds. S. Duplij and J. Wess, (Kluwer. Publ., 2001), p. 401.

20. D. D. Dimitrijevic, G. S. Djordjevic and Lj. Nesic, Fortschr. Phys. 56, No. 4-5, 412 (2008).

21. I. M. Gel'fand, M. I. Graev and I. I. Pyatetskii-Shapiro, Representation Theory and Automorphic Functions, (Saunders, London, 1966).

22. G. S. Djordjević and B. Dragovich, Mod. Phys. Lett. A 12, 1455 (1997).

23. B. L. Cherchiai, S. Schraml and J. Wess, Int. J. Mod. Phys. B 13, 3049 (1997).

24. L. Mezincesku, Star operation in Quantum Mechanics, hep-th/0007046.

25. G. Dito, D. Sternheimer, IRMA Lectures in Math. Theoret. Phys., vol. 1, 9 (2002).

26. L. C. Biedenharn, J. Phys. A: Math. Gen. 22, L873 (1989).

27. M. Gerstenhaber and D. S. Schack: Algebraic Cohomology and Deformation Theory, in Deforamtion Theory of Algebras and Structures and Applications, eds. M. Hazewinkel and M. Gerstenhaber, (Kluwer, Dordrecht 1988) pp. 13-264.

28. G. S. Djordjevic and Lj. Nesic, Towards Adelic Noncommutative Quantum Mechanics, in Particle Physics in the New Millennium; Proc. 8th Adriatic Meeting Dubrovnik, eds. J. Trampetic and J. Wess, (CRM Springer Lect. Notes in Physics), 616 (2003), p. 25.

29. J. Wudka, Phys. Rev. D 35, 3255 (1987).

30. F. Darabi and H. R. Sepangi, Class. Quantum Grav. 16, 1565 (1999.

31. F. Darabi, A. Rezai-Aghdam, A. R. Rastkar, Phys. Lett. B 615, 141 (2005).

32. G. S. Djordjevic, Lj. Nesic and D. Dimitrijevic, Notes on Ultrametric Extra Dimensions and Noncommutative Quantum Cosmology, in Strings@ the Black Sea, eds. P. P. Fiziev and M. D. Todorov, (St. Kliment Ohridski University Press, Sofia, 2006), p. 173.

33. D. D. Dimitrijevic, G. S. Djordjevic and Lj. Nesic Journal of Research in Physics, Vol. 31, No. 2, 122 (2007).

34. A. Zejak and B. Dragovich Noncommutative Minisuperspace Cosmology, arXiv: 0708.3950 .

35. R. Horvat and J. Trampetic, Phys. Lett. B 710, 219 (2012). 